\title{
Simulador cirúrgico e realidade virtual no ensino de cirurgia de catarata
}

A

realidade virtual é o uso de diversas tecnologias digitais para criar a ilusão de uma realidade que não existe de verdade, fazendo a pessoa mergulhar em mundos criados por um as suas primeiras aplicações, destacam-se os simuladores de voo, instrumentos fundamentais no treinamento de futuros pilotos. Ainda hoje, muitos acreditam que a realidade virtual só tem aplicação no entretenimento, em jogos eletrônicos e parques de diversão. Na verdade, ela tem aplicações práticas importantes e vem se expandindo em diferentes áreas como educação, com ensino à distância, indústria, no treinamento de pessoas para atividades de risco, e, principalmente, na Medicina, onde já é utilizada em tratamentos de transtorno do pânico e outras fobias, em teleconferências e, mais recentemente, no aprimoramento e desenvolvimento de novas técnicas cirúrgicas.

A realidade virtual tem potencial de aplicação em qualquer circunstância na qual ensaios repetidos são necessários para aquisição de habilidades. Na oftalmologia, o uso de simulador com realidade virtual para ensino de cirurgias intraoculares já é REAL.

Na última edição da Revista Brasileira de Oftalmologiafoi publicado o artigo de revisão "Oftalmologia e realidade virtual"(1), que abordou aspectos importantes da realidade virtual e suas diversas aplicações na área médica, e gostaríamos de acrescentar como o uso de simuladores tem sido utilizado no ensino de cirurgia intraocular em diversos centros de referência de ensino do mundo, tendo recentemente chegado ao Brasil. Dentre os simuladores mais conhecidos estão: Eyesi, produzido por VRmagic, Alemanha, e PhacoVision, produzido por Melerit, Suécia, além de outros protótipos que estão em desenvolvimento por centros de pesquisa, como, por exemplo, "Computerbased training system for cataract surgery", em pesquisa no Instituto Nacional de Investigación en Informática y Automática de Francia (INRIA) e departamento de Oftalmologia da Universidade de Lille, França.

O simulador Eyesi, por exemplo, é utilizado em mais de 50 instituições de ensino na América do Norte e Europa para treinamento de seus residentes em cirurgia de catarata por facoemulsificação, previamente ao início das atividades cirúrgicas em pacientes e olhos reais. Os resultados deste treinamento já estão na literatura, demonstrando, por exemplo, a redução de tempo e potência de faco nas cirurgias realizadas por residentes que realizaram o treinamento, uma curva de aprendizado da técnica cirúrgica mais acelerada e menos complicações ${ }^{(2)}$.

O aparelho consiste de um sistema com microscópio binocular montado em mesa com cabeça e olho artificiais acoplados a computador, pedal do microscópio e do facoemulsificador, reproduzindo muito fielmente o equipamento cirúrgico. A elasticidade e resistência dos tecidos intraoculares na realidade virtual (córnea, íris, cápsula, núcleo, córtex) têm semelhança de comportamento com os tecidos reais sufuciente para possibilitar o desenvolvimento e aprimoramento das habilidades necessárias à realização da cirurgia "in vivo". O equipamento possui critérios de avaliação do desempenho do aluno com feedback imediato e sequência de exercícios com graus de dificuldade crescentes.

Desde 2003 há artigos na literatura científica descrevendo o seu uso no treinamento e desenvolvimento de técnicas de vitrectomia via pars plana e de cirurgia de catarata $^{(3-6)}$. Sua aplicação como método de ensino para facoemulsificação inclui as etapas de capsulorrexe, hidrodissecção e delineação, emulsificação do núcleo e aspiração do córtex e já foi documentado e validado ${ }^{(7-11)}$.

O treinamento prático com simuladores cirúrgicos (que vem sendo denominado "Dry-lab", em oposição ao tradicional "Wet-lab", onde são utilizados olhos de animais para o treinamento ex- 
perimental) já é realidade no Brasil. O simulador Eyesi foi utilizado pela primeira vez no Brasil, com seu módulo de retina, no "Curso Avançado de Retina", realizado no Rio de Janeiro, em outubro de 2011, pelo Curso de Pós-Graduação em Oftalmologia da Escola Médica da PUC-RJ, sob Coordenação do dr. Flávio Attanasio de Rezende, Professor associado da PUC-RJ e Universidade de Montreal, Canadá, onde o equipamento já é utilizado como rotina para treinamento de seus residentes. Em abril de 2012, o equipamento começou a ser utilizado no Rio de Janeiro, no "Curso de treinamento prático em cirurgia de catarata com simulador cirúrgico Eyesi”, sob coordenação do dr. Flávio Rezende, Professor Instituto Nacional de Investigación en Informática y Automática de Francia (INRIA) e Titular de Pós-Graduação em Oftalmologia da PUC-RJ.

Alguém, hoje, entregaria um avião ou sua vida a um piloto que se inicia na arte de conduzir pelos ares pela primeira vez sem que tenha antes realizado horas e horas de treinamento em um simulador? Da mesma forma acreditamos, como profissionais envolvidos na formação de médicos oftalmologistas, que o uso dos simuladores cirúrgicos inicia uma nova era na educação médica e no treinamento dos residentes, poupando pacientes das mãos inexperientes dos alunos e reduzindo os riscos de complicações e traumas físicos e psicológicos, tanto para paciente, como para o aluno e professor, em uma época em que a exigência de melhores resultados é crescente.

Flavio Rezende', Renata Attanasio de Rezende Bisol ${ }^{1}$, Tiago Bisol', Flavio Attanasio de Rezende ${ }^{1,2}$

'Pontifícia Universidade Católica do Rio de Janeiro - PUC-RJ - Rio de Janeiro (RJ), Brasil.

${ }^{2}$ Universidade de Montreal, Canadá.

\section{REFERÊNCIAS}

1. Carvalho JA. Oftalmologia e realidade virtual. Rev Bras Oftalmol.2012;71(1):40-7.

2. Belyea DA, Brown SE, Rayyoub LZ.Influence of surgery simulator training on ophthalmology resident phacoemulsification performance. J Cataract Refract Surg. 2011;37(10):1756-61.

3. Jonas JB, Rabethge S, Bender HJ. Computer-assisted training system for pars plana vitrectomy. Acta Ophthalmol Scand. 2003;81(6):600-4.

4. Rossi JV, Verma D, Fujii GY, Lakhanpal RR, Wu SL, Humayun MS, et al.Virtual vitreoretinal surgical simulator as a training tool. Retina.2004;24(2):231-6.

5. Grimm J. Tearing of membranes for interactive real-time surgical training. Stud Health Technol Inform. 2005;111:153-9.

6. Webster R, Sassani J, Shenk R, Harris M, Gerber J, Benson A, et al.Simulating the continuous curvilinear capsulorhexis procedure during cataract surgery on the EYESI system. Stud Health Technol Inform.2005;111:592-5.

7. Mahr MA, Hodje DO. Construct validity of anterior segment anti-tremor and forceps surgical simulator training modules: attending versus resident surgeon performance.J Cataract Refract Surg. 2008;34(6): 980-5.

8. Feudner EM, Engel C, Neuhannm IM, Petermeier K, Bartz-Schmidt KU, Szurman P. Virtual reality training improves wet-lab performance of capsulorhexis: results of a randomized, controlled study. Graefes Arch Clin Exp Ophthalmol.2009;247(7):955-63.

9. Solverson DJ, Mazzoli RA, Raymond WR, Nelson ML, Hansen EA, Torres MF,et al. Virtual reality simulation in acquiring and differentiating basic ophthalmic microsurgical skills. Simul Healthc.2009;4(2):98-103.

10. Privett B, Greenlee E, Rogers G, Oetting TA. Construct validity of a surgical simulator as a valid model for capsulorhexis training. J Cataract Refract Surg. 2010;36(11):1835-8.

11. Le TD, Adatia FA, Lam WC.Virtual reality ophthalmic surgical simulation as a feasible training and assesment tool: results of a multicentre study. Can J Ophthalmol.2011;46(1):56-60.

\footnotetext{
Endereço para correspondência:

Rua 19 de Fevereiro, 76

Botafogo - Rio de Janeiro - RJ

CEP: 22280-030
} 